\title{
Direction and trend of networking technology in Japan to save finite natural resources - after undergoing the East Japan megaquake
}

\begin{abstract}
Akira Arutaki
Network Design Research Center, Kyushu Institute of Technology, 680-4 Kawazu, Iizuka, Fukuoka 820-8502, Japan

Email: aarutaki@ndrc.kyutech.ac.jp

Abstract: The East Japan megaquake left huge impacts to the entire nation. The information and communication technology area is not an exception. The destruction of nuclear power plants by the tsunami led the poverty in electric power, which forced the Japanese people to see energy saving in a new light. The confusion in telecommunication, caused by the destruction of the networks and the congestion of heavy emergency traffic, also amplified the necessity of efficient utilisation of finite radio frequencies. This paper introduces the direction and trends of R\&D activities in Japan for saving natural resources such as energy and radio frequency, which are focused more intensively after the disaster. 'Green' activities try to expand their contribution to the telecommunication area. Many smart grid projects have been accelerated and have started field trials. New schemes to share limited frequencies also came into the spotlight. These activities will save natural resources more effectively than ever.
\end{abstract}

Keywords: the East Japan megaquake; information and communication technology; ICT; natural resource saving; power consumption; smart grid; smart metre; smart city; cognitive radio; frequency sharing; mesh network; Japan.

Reference to this paper should be made as follows: Arutaki, A. (2016) 'Direction and trend of networking technology in Japan to save finite natural resources - after undergoing the East Japan megaquake', Int. J. Soft Computing and Networking, Vol. 1, No. 1, pp.35-54.

Biographical notes: Akira Arutaki received his BE and ME degrees from Tohoku University in 1978 and 1980, respectively, and PhD from Kyushu Institute of Technology in 2008. Joining NEC Corporation in 1980, he was engaged in research and development of digital communication systems. From 1987 to 1990, he stayed at Washington University in St. Louis, MO, as a scholarship recipient. He was the General Manager of System Platforms Research Laboratories from 2003 to 2006, and was assigned to Associate Senior Vice President of NEC Communication Systems, Ltd. in 2006. He joined Kyushu Institute of Technology as a Professor in 2009. He received the Network Systems Research Award of IEICE in 1999, and won the Incentive Award of Japan Institute of Invention and Innovation in 2013. He is the author of three books in the packet communication field. He is a member of IEEE and IEICE of Japan. 


\section{Introduction}

The information and communication technologies (ICTs) have been recognised as the bases of national industrial foundation in Japan. The recent research and development activities of ICT are conducted by IT companies as well as by universities and government agencies. These activities range from hardware to software, such as post LTE, software defined network, big data, etc. This trend is much the same as for other countries' R\&D activities in the world. One peculiarity lies in the natural circumstances of Japan. Natural resource saving area is one of the most important fields for Japanese R\&D activities because of large population and narrow land size of the country.

For example, this importance is led by the energy balance (The Federation of Electric Power Companies, 2011) as shown in Figure 1. Japan is originally one of the most import-excess countries in terms of natural resources. Self-sufficiency in energy was only $18 \%$ in 2008 . Therefore, Japanese R\&D organisations have been focusing on 'how to overcome the lack of natural resources'. In addition, the East Japan megaquake in 2011 gave drastic stimulations to this trend.

Figure 1 Energy balance of the major countries

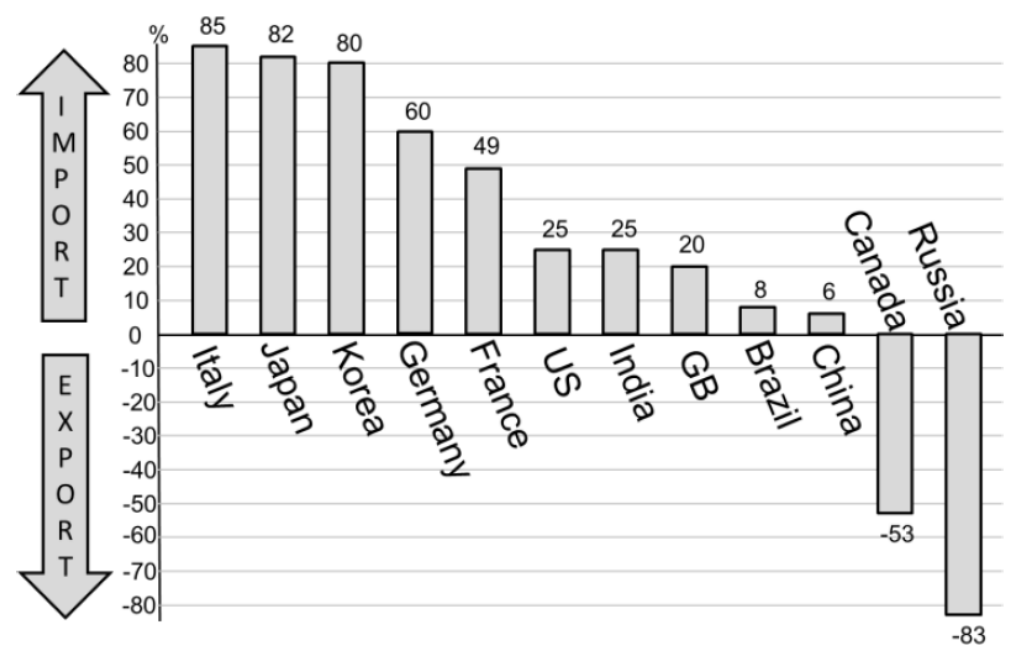

Source: The Federation of Electric Power Companies of Japan

Right after the megaquake, the huge tsunami attacked the coast area, which was higher than three-story buildings; Figure 2 pictures the tsunami and an evacuee on the roof of a four-story building. Some of the nuclear power plants were also attacked, and eventually the reactors exploded. This accident forced the Japanese government to stop all the nuclear power plants in Japan. 'To restart them, or not to do?' National arguments were caused, and have never reached the conclusion yet. As a natural result, the $\mathrm{coal} /$ petroleum/liquefied natural gas (LNG) import for thermal power plants has been increasing. Then the self-sufficiency in energy in Japan decreased to 6\% (KEIDANREN, 2013) from $18 \%$, and the trade balance turned into deficits after the disaster.

To cope with this situation, many activities were planned and have been conducted. Some green ICT projects try to accelerate renewable energy deployment. Other projects try to reduce electricity consumption of ICT equipment. Smart grid is another attempt to 
construct efficient electric power systems. This paper introduces some R\&D activities of green ICT and smart grid in Japan with respect to saving natural resources.

Figure 2 The tsunami and an evacuee

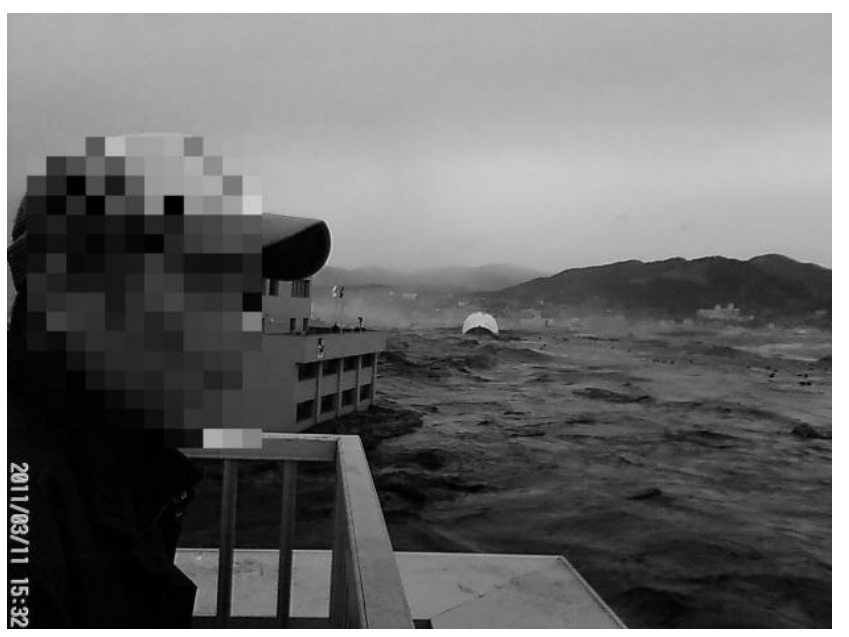

The radio frequency (RF) is another natural resource which is not infinite. Recent penetration of cellular and smart phones has led to heavy traffic in the air. According to the recent survey (NTT Data Institute of Management Consulting, Inc., 2013), it is estimated that the cellular and Wi-Fi traffic in Japan will be more than 40 times larger in 2025 than that of 2011 as shown in Figure 3(a). However, the frequencies in very high frequency (VHF) and ultra high frequency (UHF) bands are almost completely/all licensed, i.e., no vacancy in the bands. This forecast urges researchers to accelerate their activities of cultivating new RF channels.

Figure 3 Background for more flexible utilisation of frequencies, (a) wireless traffic explosion (b) damages of cellular systems by megaquake*

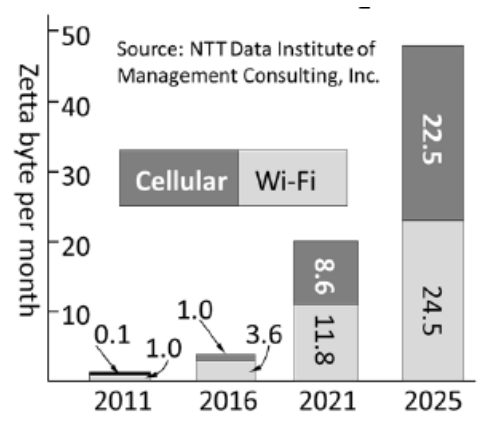

(a)

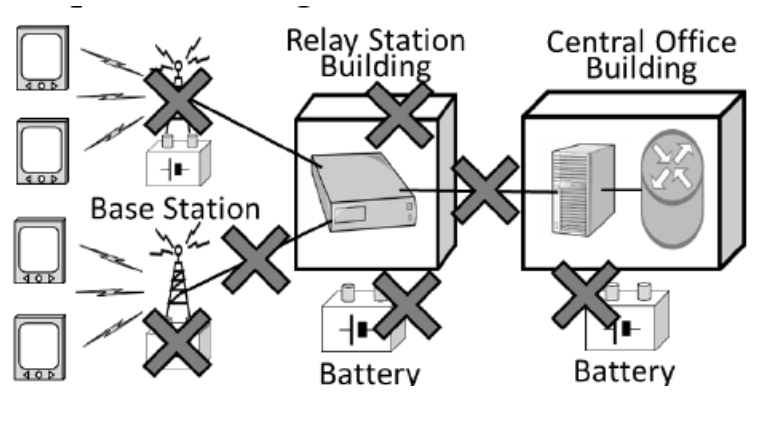

(b)

Source: *Ministry of Internal Affairs and Communications

Another concern for frequencies is disaster recoveries. It is reported in the white paper (Ministry of Internal Affairs and Communications, 2011) that the megaquake brought serious damages to the public communication networks as depicted in Figure 3(b). Many 
base stations, relay stations, and central offices were destroyed as well as transmission lines, which are indicated by ' $\mathrm{X}$ ' in Figure 3(b). According to the white paper, huge number of voice calls were initiated after the megaquake, which generated up to 60 times heavier cellular traffic than usual. As a result, the traffic congestion forced the carriers to restrict call-origination to keep the network stable. This phenomenon teaches us to provide more flexible wireless communication systems for disaster areas to recover quickly. Cognitive radio and mesh networking are expected to establish prompt backups as effective frequency-saving technologies. This paper also covers these technologies researched in Japan.

\section{Green ICT}

The East Japan megaquake left huge impacts everywhere in the country as well as in the disaster areas. One of them is a green curtain boom. As is shown in Figure 4, many households and companies became to plant greens in front of the windows to get shade for less electricity consumption during summertime following the government suggestions (Suzuki, 2012). As a matter of fact, the public opinion survey after the disaster indicated that almost $60 \%$ of entire Japanese citizens started trying to intensively save electricity (Cabinet Office, Government of Japan, 2012) as shown in Figure 5. This awareness dominated the other opinions such as the preparation against disasters.

Figure 4 Green curtain

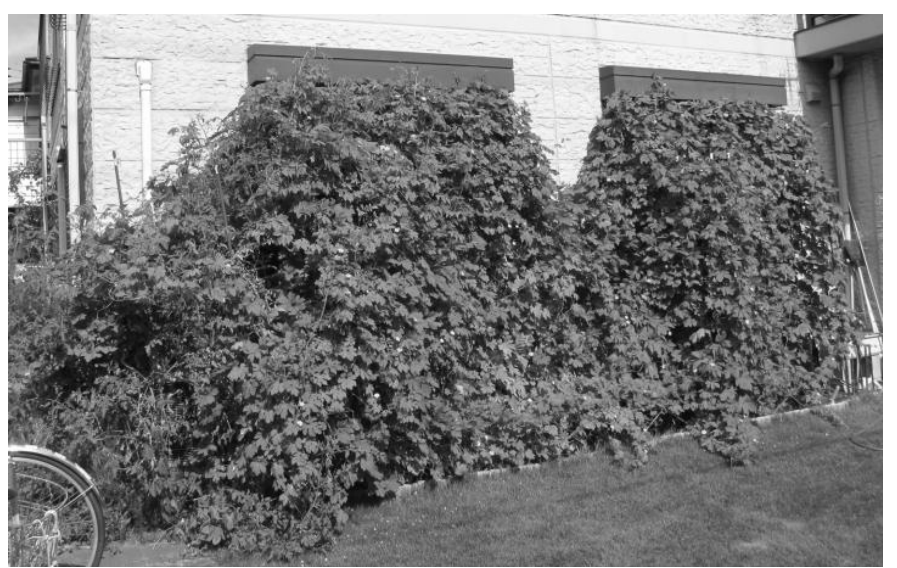

Renewable energy, such as solar, wind, tidal, and geothermal power, is expected to contribute for the future earth-friendly energy infrastructure. Ministry of Economy, Trade and Industry of Japan (MITI) is promoting solar power generation to increase 40 times larger in 2030 compared to that of 2005. However, this approach takes time because of requiring a huge amount of public and private investment. Therefore, other approaches to save energy need to be applied for today's solution. 
Figure 5 Public opinion survey

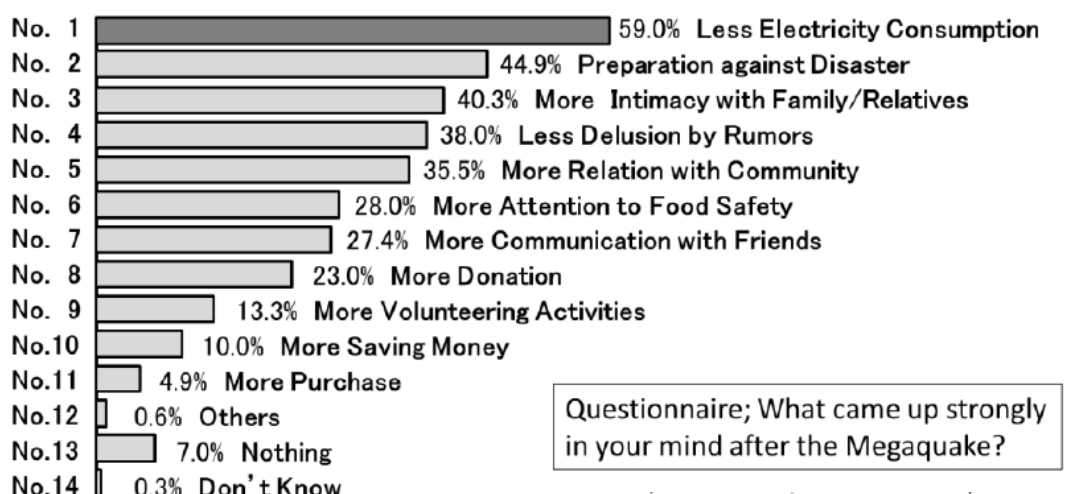

Source: Cabinet Office, Government of Japan

\subsection{Top runner program}

Agency for Natural Resources and Energy, one of the organisations in METI, has started 'Top runner program' (Kimura, 2010) in 1998, by which energy-saving equipment, such as appliances, is certificated. A machine with this certification can be decorated by 'Top runner sticker' on it. This programme promotes to develop energy-saving equipment. Routers and switches for networking were nominated to this programme in 2011.

Readers may assume that the total amount of power consumption by routers and switches in Japan is negligibly small compared to other devices, such as motors, lighting, air conditioners, etc. According to the survey (Ministry of Internal Affairs and Communications, 2008) by the Government of Japan, it is far from negligible smallness. This survey forecasted that $6.4 \%$ of entire electric power in Japan in 2012 would be consumed by the ICT, such as the internet. Furthermore, it was also forecasted that the power consumption by routers and switches would be in the similar range, specifically speaking, more than half of the electricity consumption by PCs, servers and storage devices as depicted in Figure 6.

Figure 6 Electric power consumption forecast by ICT in 2012

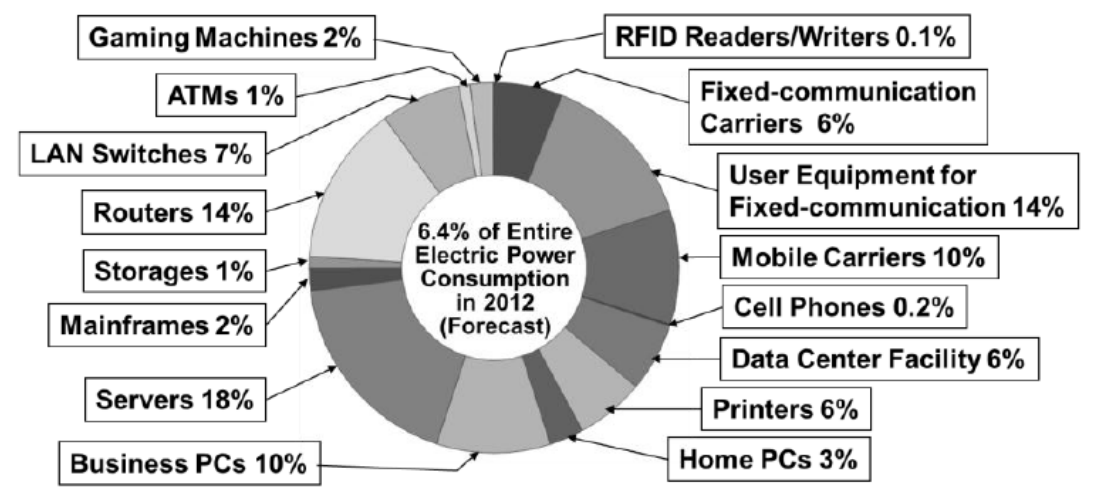

Source: Ministry of Internal Affairs and Communications 
The longer-term forecast is reported with respect to the major ICT devices; router and switches, servers, storage devices and PCs (NTT Data Institute of Management Consulting, Inc., 2013). As is shown in Figure 7, assuming that no improvement of power saving technologies is applied, the power consumption of routers and switches for networking in 2025 and 2050 will dominate the others'. Therefore, it is concluded that power elimination of networking devices is one of the keys for future resource saving.

Figure 7 Power consumption forecast of major ICT devices

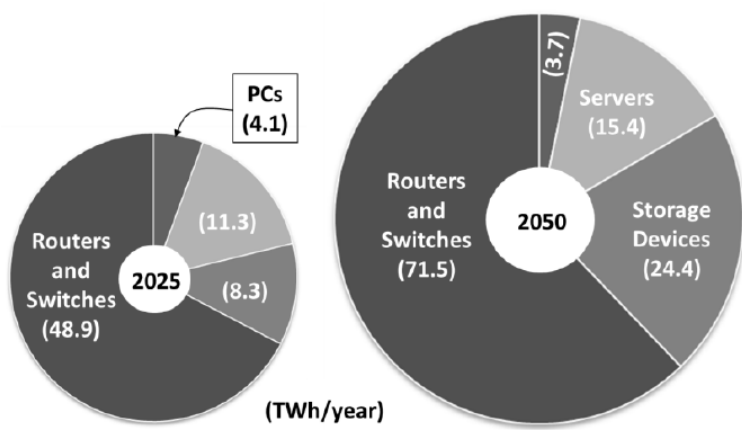

Source: NTT Data Institute of Management Consulting Inc.

\subsection{For less power consumption of networking equipment}

One of the power-saving technologies is, as is well-known, to let servers (CPUs) and storage devices sleep, when they are not working. It is unfortunate that this idea of partial-sleeping has not been implemented into line-interface cards (IF) accommodated in networking devices such as routers, etc. This is because it is not easy to let two IFs at each end of the transmission line sleep/wake up at the same time. If they sleep at the same time, they cannot wake the other up, and keep sleeping forever. If some implementation can support the above 'partial sleep' function for IFs accommodated in large-scale routes/switches, it will perform strong energy saving, because the larger networking devices consume the more relative power of IFs.

Figure 8 illustrates a typical architecture of networking devices. Networking devices 1 and 2 are connected via four transmission lines in the illustration as an example. Ordinary networking devices are not furnished with the power control mechanisms for IFs, hence, all IFs in the devices are always fed with electricity and consume it regardless of the traffic amount through them. If electric power fed to an IF were controlled, which conveys light traffic, unnecessary power feeding would be suppressed by forcing the IF to sleep. It is needless to say that the light traffic via the IF needs to be bypassed to other transmission lines prior to the power control of the IF.

A new technology (Fukuda et al., 2012) provides the practical solution to this issue by taking advantage of the link aggregation control protocol (LACP) (IEEE, 2000), and realises the power-saving implementation. The original purpose of LACP is to sprit high-speed traffic for utilising multiple slower physical links, and to aggregate these multiple low-speed streams of traffic to the original high-speed one. This protocol is also used to isolation faulty links. For these purposes, LACP identifies the mapping of physical connections (i.e., physical links) and logical streams (i.e., logical links). 
Figure 8 Networking device architecture

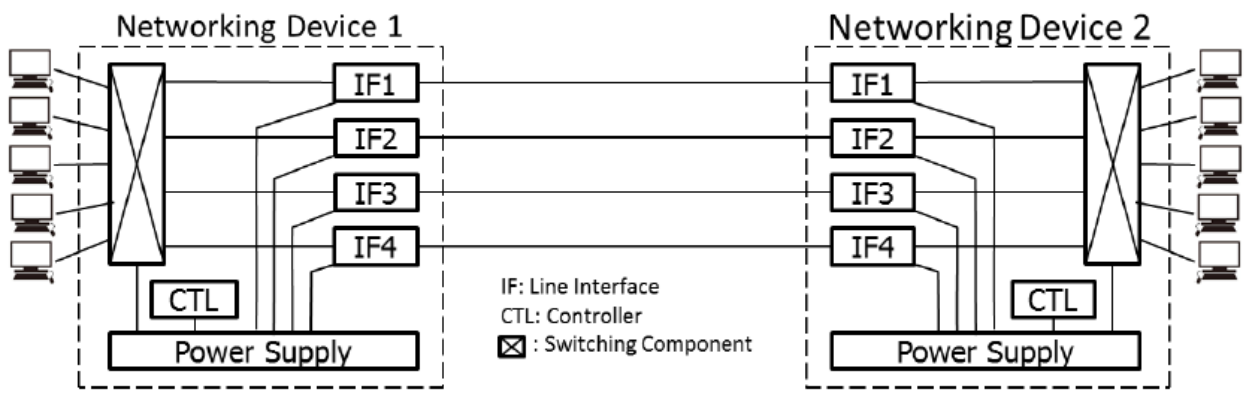

Figure 9 Power saving scheme by lulling line interfaces to sleep

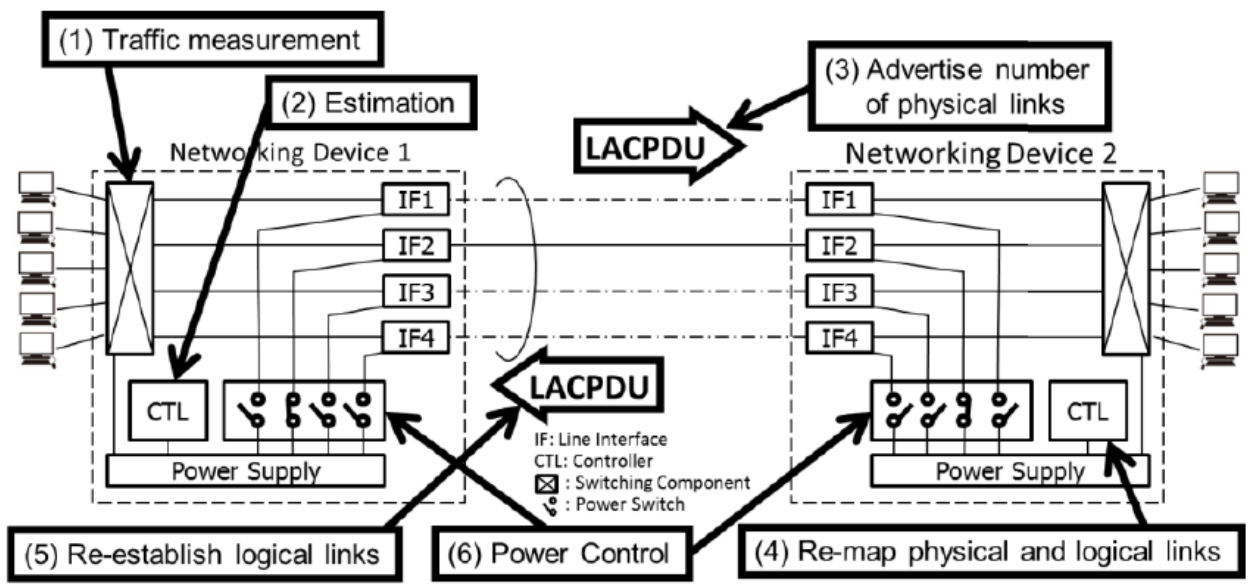

Figure 9 illustrates the mechanism to utilise LACP for the purpose to reduce the power consumption of IFs. As an example of this illustration, the networking devices 1 and 2, such as routers, are connected by four physical transmission lines (trunks) which are terminated by IF1 to IF4 in the devices. Each network device accommodates five subscribers, such as servers, at the opposite side of the trunks. The power supply feeds necessary electricity to all components in the network devices, i.e., to the IFs, controller (CTL) and switching component.

The new scheme of power saving needs some additional features of the network device for taking advantage of LACP; one is to measure the entire traffic which the device conveys, and the other to estimate necessary bandwidth in the trunks. Adding these features, the power-saving operation is as follows (refer to Figure 9):

1 Traffic from/to the subscribers is measured at the switching component in the network device 1 .

2 The controller in the network device 1 estimates how much bandwidth is necessary to convey the above traffic via the trunks.

3 The controller sends LACPDU (link aggregation protocol data unit) to advertise how many trunks are necessary to convey the traffic. 
4 The controller in the network device 2 receives the LACPDU, and re-maps the logical links to the minimum number of physical links which have enough capacity to convey the traffic.

5 Then it sends LACPDU to the network device 1 to re-establish the logical links.

6 When the negotiation between the network devices 1 and 2 completes, power switches in both network devices are set to lull IFs to sleep, which are not supposed to convey the traffic.

Note that, as an example in Figure 9, two IF2s connected by the solid line are fed with power, and are awake. The other dash-dot lines connect the sleeping IFs which are not powered. Also note that the practical design of the power switch does not adopt just a mechanical contact but more sophisticated mechanism to cut the power feeding to the IFs.

The authors of this paper (Fukuda et al., 2012) confirmed that this scheme works properly, i.e., the power consumption of the line cards in the router varies proportionally to the traffic to be conveyed. Their experiment system is pictured in Figure 10(a). It is observed that the power consumption varies linearly depending on the traffic volume as shown in Figure 10(b).

Figure 10 Experiment of the new mechanism, (a) experiment system (b) performance evaluation (see online version for colours)

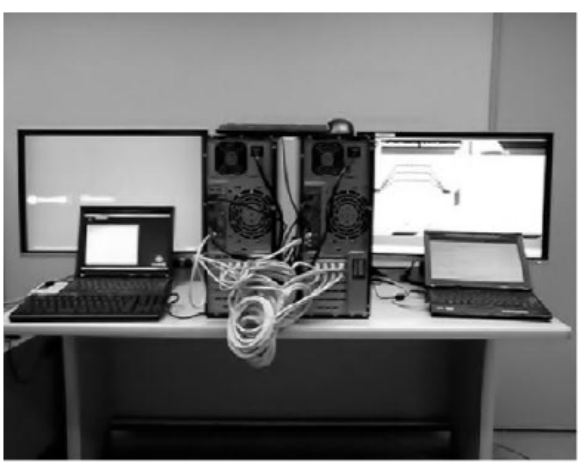

(a)

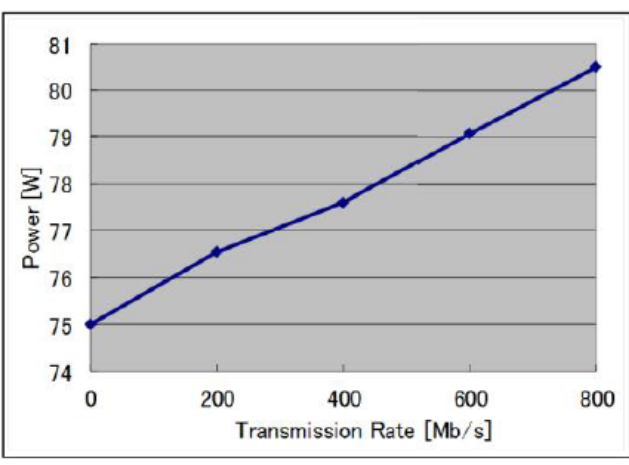

(b)

\section{Smart grid}

As is mentioned in the earlier section, the megaquake left the huge impacts to electric power provision and consumption in Japan. For good or for evil, these impacts accelerated the development and deployment activities of the smart grid. This section covers recent topics of the smart grid in Japan.

\subsection{Quick overview of the electric power grid in Japan}

Figure 11 depicts a quick overview of the electric grid in Japan (Chubu Electric Power Company, http://www.chuden.co.jp/ryokin/information/chishiki/mame_hatsuden/index. 
html). This illustration analogises to the grid of the USA for the explanation purpose (Brown and Sedano, 2004). It is understood that the voltage in generating stations and transmission lines is relatively low compared to the other countries. This is because the land size of Japan is small. The distribution and consumption systems are similar to the other countries. Most of the households are fed with single-phase AC200V which is divided into $\mathrm{AC} 100 \mathrm{~V}$ at a fuse box at home.

Figure 11 Electric Grid in Japan

Source: Chubu Electric Power; Brown, M (2004)

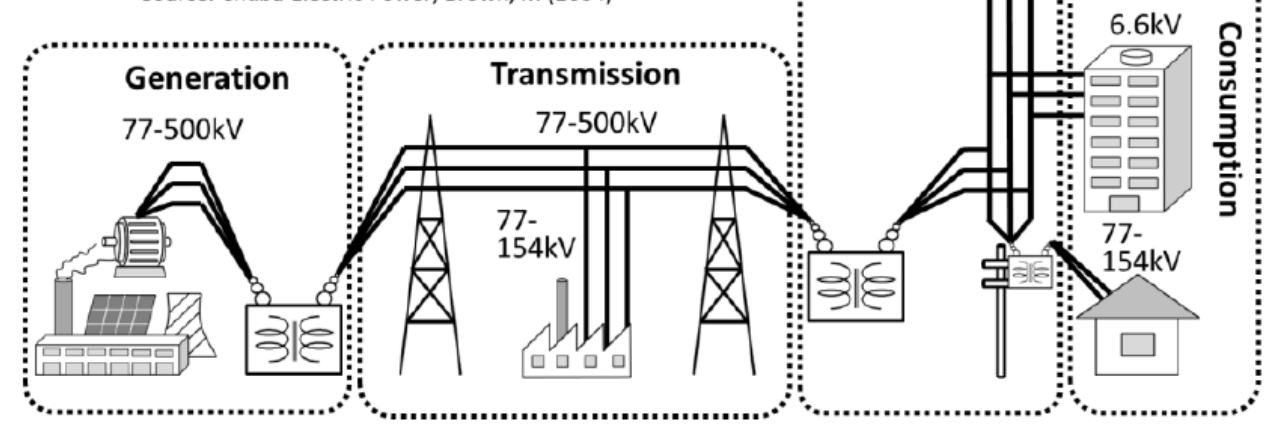

Figure 12 Total duration of power cuts [minutes/year per user], (a) comparison of major countries (b) improvement of power-cut in Japan

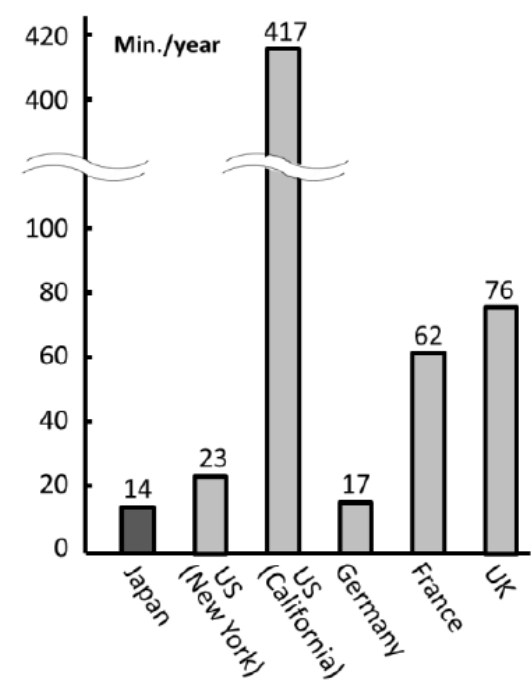

(a)

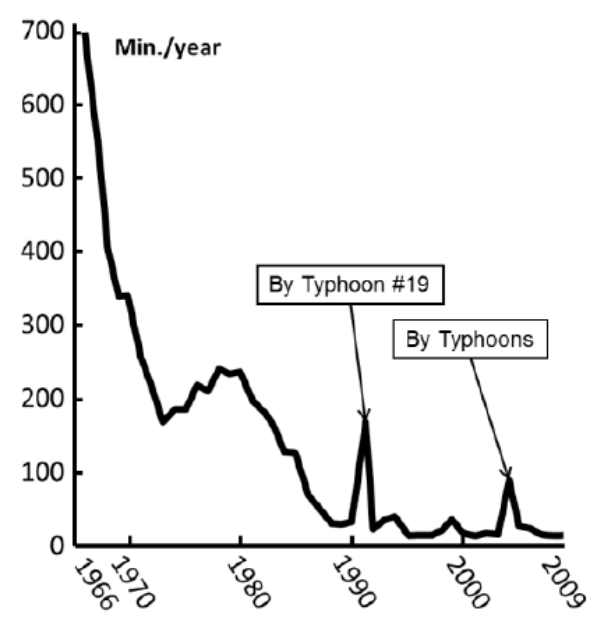

(b)

Source: The Federation of Electric Power Companies 
This grid has been strategically designed to keep stable and reliable power supply, not a patch work. As a natural result, the supply quality of electricity in Japan is better than the other countries as shown in the chart of Figure 12(a). The quality has been improved by continuous innovation of the grid system. The chart of Figure 12(b) depicts its history since the mid 60s (The Federation of Electric Power Companies, 2010). The power-cut duration per user became almost $1 / 50$ during the period. This fact implies that the smart grid deployed in Japan is required to keep this quality.

Figure 13 Electric power companies in Japan

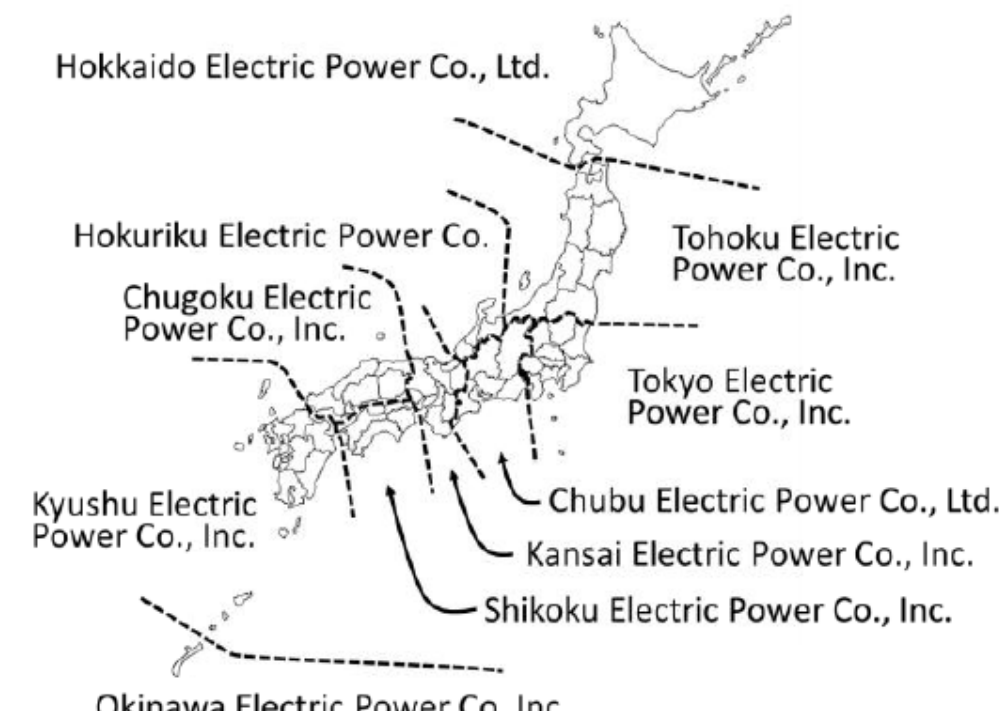

Source: The Federation of Electric Power Companies

Figure 14 Generation amount of electric power

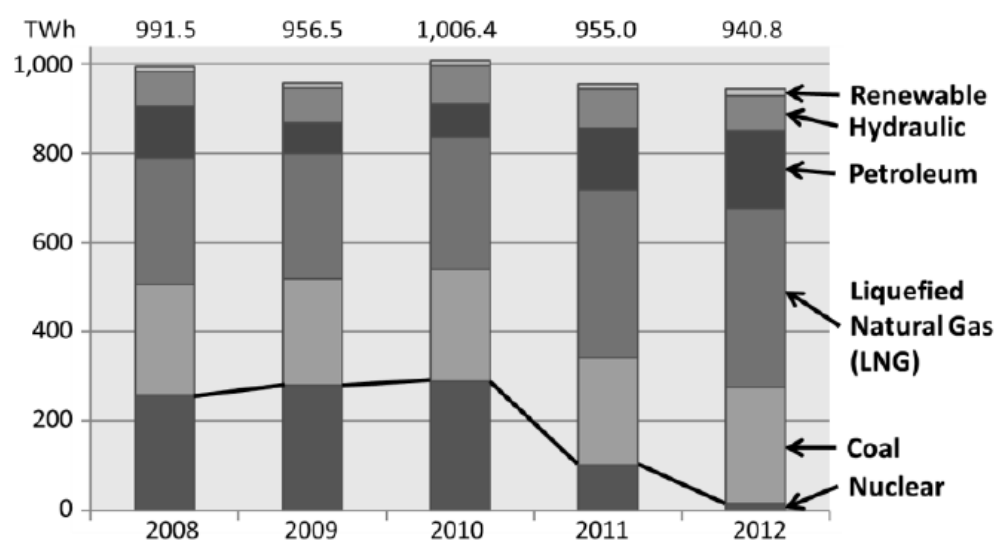

Source: The Federation of Electric Power Companies 
There are ten electric power companies and one wholesale electricity utility in Japan as shown in Figure 13. They are supervised by the government, but not monopolised. More than half of their generating stations are on a thermal power generation basis. Nuclear power generation had been increasing and was assumed to be dominant in the future before the megaquake. But as is mentioned previously, all the nuclear power reactors were stopped gradually after the disaster. Then the generation amount decreased as is indicated in Figure 14 (The Federation of Electric Power Companies, 2013), and eventually fossil fuel import has been increasing to compensate the nuclear loss. These facts are accelerating the power-saving activities explained in the previous section as well as smart grid deployment.

\subsection{Smart grid in Japan}

Ministry of Economy, Trade and Industry (2010) presented a plan to introduce smart grid into households and buildings first, and then to regional areas. It also aims gradual and eventual penetration of smart grid to the entire nation; namely, the entire nation will be covered with wide-area smart grid by connecting the regional-area ones as depicted in Figure 15.

Figure 15 Smart grid deployment plan

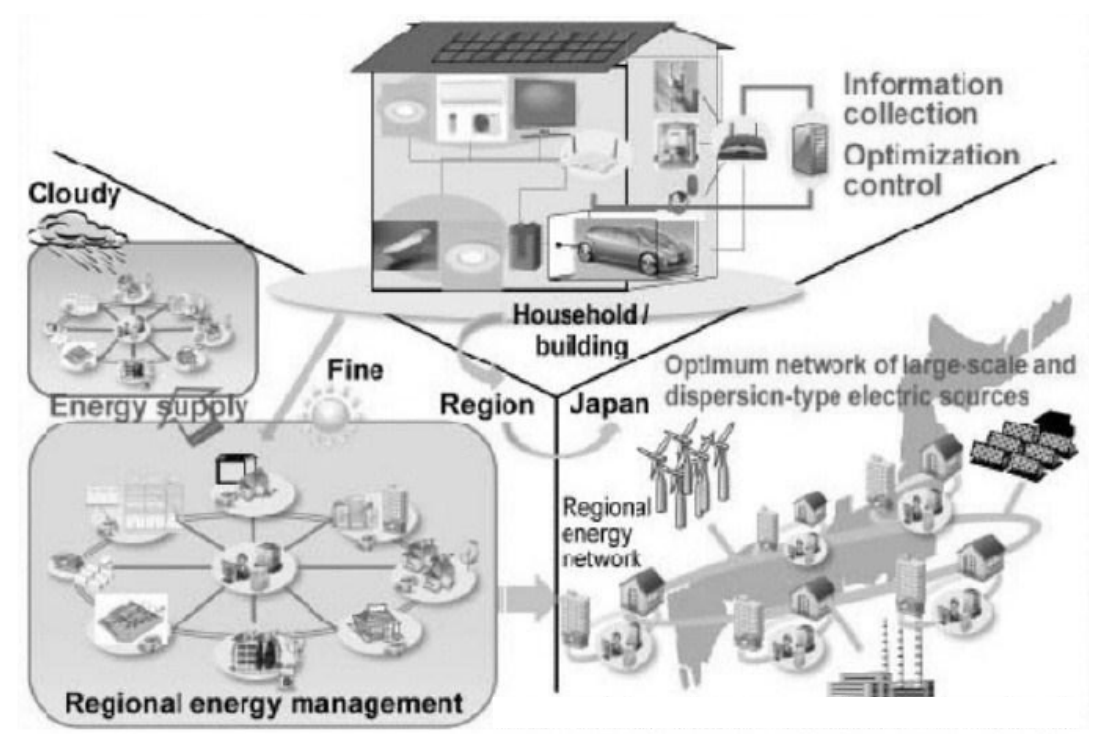

Source: Ministry of Economy, Trade and Industry (2010)

One of the original emphases of this plan is placed on the promotion of private (i.e., homes and offices) solar power generation and of introducing reflux technologies for surplus electricity by the generation. Another emphasised purpose is to monitor the electricity consumption and to save power at home/office in the first step of the plan. This direction was accelerated by the megaquake. 
Figure 16 Smart house

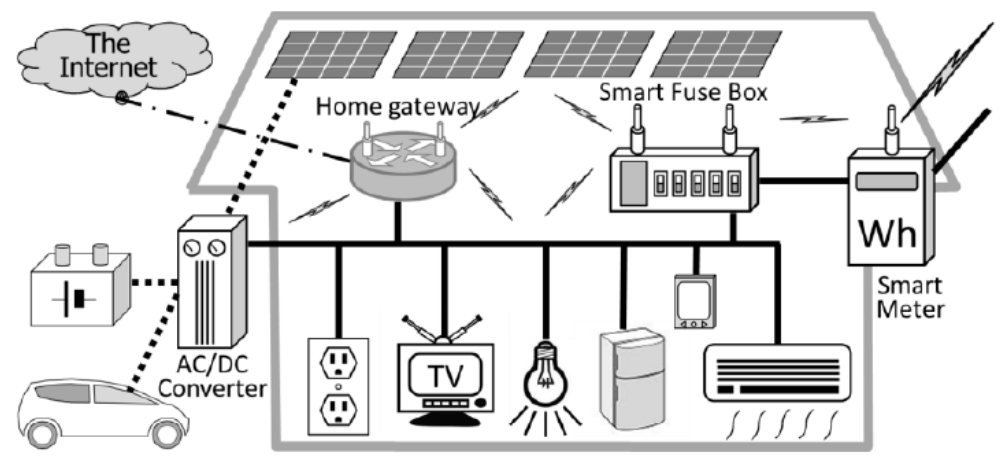

Numbers of trials (for example, Nikkan Kogyo Shimbun, Ltd., 2013) are being conducted to make sure that the smart houses are practical and effective for energy saving. These projects involve many participants; local governments, electric utility companies, general constructors, house builders, equipment vendors, real estate companies, etc. Figure 16 illustrates a typical example of the smart house configuration. It is needless to say that the smart metre furnished at home is a key for metering electricity consumption. However, it is not a single device to construct the smart house. A smart fuse box inside a house monitors and controls the entire current flow at home. All the devices and appliances are connected to this smart fuse box, and are controlled to work efficiently. Solar panels, plug-in hybrid electric vehicle (PHEV), and fixed-type batteries are also connected to the smart fuse box via AC/DC converter whose connections among them are on a DC basis; dot-dash lines in Figure 16 indicate the DC-based power lines.

Communication between the smart fuse box and in-house devices can be performed via Wi-Fi and/or power line communication (PLC). Power consumption status of in-house appliances are monitored via this communication link, and the efficient operation of the appliances are controlled by the fuse box also via this link. Communication between the smart house and central controllers in the wide area, which is typically the servers of the electric power company, is performed via Wi-Fi, cellular, optical line, etc. The smart metre on the house-wall functions as a communication device for this scheme. According to the recent press release (Tokyo Electric Power Company, 2014), Tokyo Electric Power Co., Inc. (TEPCO), the largest regional electric power company in Japan, has decided to install smart metres into all of their customer houses in 2020 despite that the all subscribers are not necessarily smart houses. Note that this plan adopts Wi-Fi and/or PLC which Ministry of Internal Affairs and Communications (MIC) has recently (March 2014) approved to operate outside of home. Note that some trials of smart house adopt home gateways to collect information of power consumption and to control appliances as depicted in Figure 16.

Another impact brought by the megaquake is to storage electricity at home. The grid in the East Japan was destroyed by the disaster, which forced people living in the area to survive without electricity more than a month at most. Batteries installed in residential site and in PHEVs are expected to be alternative power sources during the power-cut period. 
Figure 17 Smart city

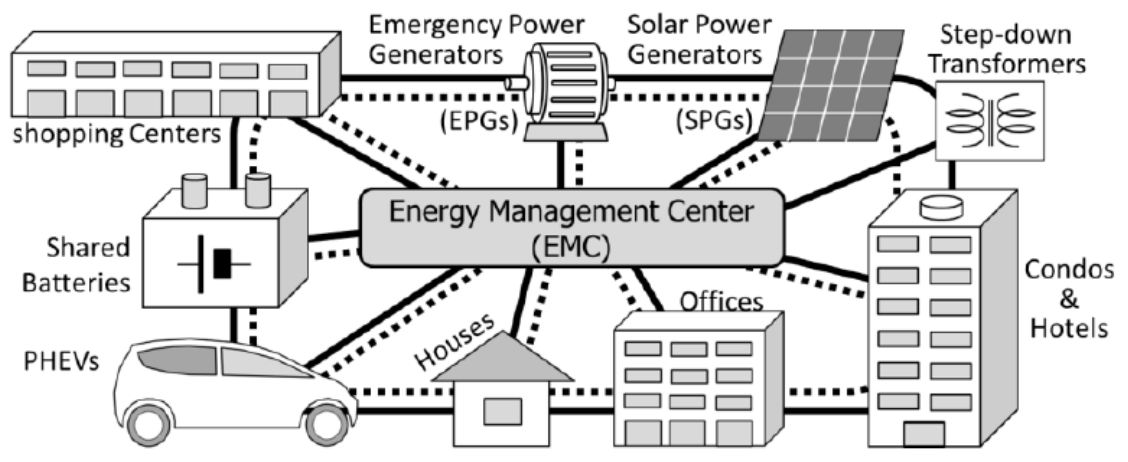

The next step of the smart grid deployment is smart city. Mitsui Fudosan Co. Ltd. (2014), one of the largest real estate companies in Japan revealed that the practical field trial of the smart city is currently operating in a suburb of Tokyo. Figure 17 illustrates the typical configuration model of smart city as an example. The energy management centre (EMC) is connected to all subscribers in the community including solar power generators (SPGs), emergency power generators (EPGs) and shared batteries. The primary distribution grid, which the solid lines indicate in Figure 17, is connected to transmission grids via the step-down transformers, and is used for normal operation. This grid is shut down in an emergency, and instead, the backup grid shown in dotted lines in Figure 17 substitutes for the primary grid. In this case, EPGs and SPGs are the power sources which may not be able to feed full power to all the subscribers. The EMC controls and restricts the current flows to keep the backup grid stable.

The final stage of METI's plan is to construct the nation-wide smart grid as illustrated in Figure 15. This stage is for further study for the future implementation.

\section{Saving frequencies}

The cellular and Wi-Fi traffic in Japan is increasing rapidly to become more than 40 times heavier in 2025 compared to that in 2011 as it was mentioned in the earlier section. On the other hand, almost all bands in VHF and UHF have already been monopolistically licensed. Therefore, it is an urgent issue to find out new channels to accommodate the increasing traffic. Additionally, one of the lessons from the damages by the megaquake is to utilise limited radio frequencies which are to be shared by as many users as possible at the same time. It is necessary to research and develop innovative technologies to perform flexible channel-sharing.

One of the solutions for the above issues is to cultivate new radio bands. Figure 18 depicts the electromagnetic frequency spectrum. As amateur radios did so more than a century ago, who found out that high frequency (HF) band was usable for long distance telecommunications after being kicked out from low frequency (LF) and medium frequency (MF) bands (ARRL, 1989), super high frequency (SHF) band and above have been researched for more services, i.e., Terahertz technologies (Teranishi et al., 2012). The current R\&D activities are in the stage of basic device development, so it is assumed that the practical solution needs more time to be utilised. 
Figure 18 Radio (electromagnetic) frequency spectrum

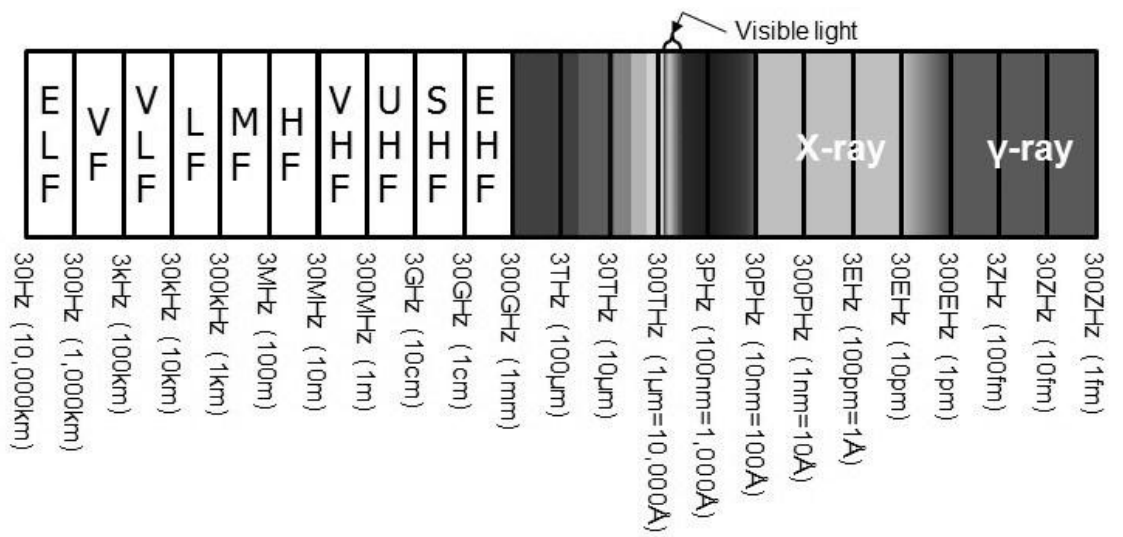

\subsection{Cognitive radio}

It is desired that a new possible solution for flexible utilisation of frequencies is devised, which reorganises the band plan and licensing schemes of VHF and UHF bands. This is because it is observed that even a licensed frequency is not necessarily always used (Ministry of Internal Affairs and Communications, 2010). Even in the central Tokyo area, except for the broadcast bands, most of licensed frequencies are not necessarily fully used. If these licensed frequencies are efficiently taken advantage of by non-privileged secondary users, the limited frequency resources provide more services without wasting them.

A new idea is currently under discussion, and will be implemented in the near future, i.e., automatic channel sharing schemes. A wireless user, who does not have privilege to monopolise a frequency, can use the frequency as the secondary user, which is licensed to the other primary user, if and only if the secondary user does not give any interference to the primary user. In the future, more dynamic frequency-sharing technology will be widely taken advantage of. Cognitive radio technology (for example, Doyle, 2009) is the major candidate to allocate a frequency to the secondary user dynamically.

Figure 19 Cognitive radio technology, (a) basic concept (b) frequency hopping (see online version for colours)

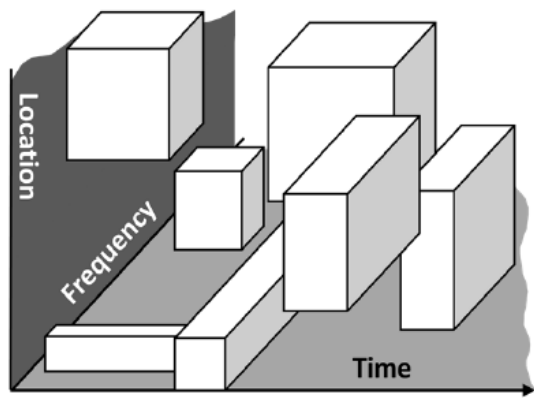

(a)

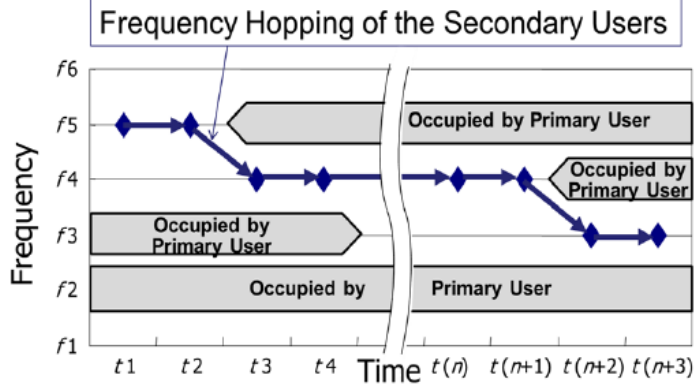

(b) 
Figure 19(a) depicts the basic concept of the cognitive radio technology. Wireless communication links can be defined by three dimensions; when, where, and what frequency. In Figure 19(a), seven wireless communication links occupy the three-dimension communication space. Each cuboid shows an occupied space by a primary user's link. The other spaces than the occupied ones are called 'spectrum holes' which can be utilised by non-privilege secondary users. Figure 19(b) illustrates how this cognitive radio communication is performed by fixed-location secondary users. Their communication link is established by using the frequency $f 5$ during the period of $t 1$ and $t 2$. They recognise the privileged primary user shows up on $f 5$, then they move to $f 4$ at the beginning of $t 3$ to give $f 5$ up, namely, frequency hopping. They operate similarly during $t(n+1)$ and $t(n+2)$ to move from $f 4$ to $f 3$. This technology is under discussion at standard-bodies such as International Telecommunication Union Radiocommunications Sector (ITU-R). Some field trials of this technology have already been conducted in Kyushu area of Japan for practical application (Altintas et al., 2012).

\subsection{Mesh networking}

It is possible to save frequency resources by running lower RF power. A typical networking configuration of cellular links is illustrated in Figure 20(a). Generally speaking in Japan, a base station for cellular communication transmits UHF electromagnetic waves of some 10 watts to cover its service area with the radius of some kilometres, depending on its location. Therefore, a frequency channel can be used by only one radio node at the same time within this service area. On the other hand, the maximum RF output-power of a Wi-Fi radio node, such as an access point, is limited to 10 milli-watts by the Japanese law (Ministry of Internal Affairs and Communications, 2013). As a result, the service area (cell) of Wi-Fi has at most a 100 metre radius depending on its location. The mesh configuration of the low-power radio nodes is illustrated in Figure 20(b), in which seven Wi-Fi nodes are connected by the low-power radio links. As the cell of each radio node is with the radius of some 10 metres, the same frequency channel can be repeatedly used by plural radio nodes in case that they are not located in the adjacent cells, i.e., more than 100 metre apart, again depending on their location. It is easily observed that the number of Wi-Fi nodes within some kilometres are much larger than that of the general cellular communication system, assuming that the bandwidth of them is equal.

One of the drawbacks of this mesh configuration is complexity and instability to control wireless links among radio nodes. The conventional way to do so adopts a broadcasting scheme. The control packet (henceforth, Hello packet) of the routing protocol for discovering and maintaining wireless links with an adjacent radio node is transmitted with broadcasting frames. Since a broadcasting frame does not have the mechanism of checking delivery, it disappears more easily by frame collisions than a unicast frame. This instability of Hello packet leads a serious situation; even if a wireless link is established, loss of Hello packets along the link leads unnecessary cut-off of the established link. Figure 21(a) illustrates this occasion. Note that ' $X$ ' denotes packet loss in the figure. Eventually, this situation causes re-establishment of an alternative link. This process induces unnecessary extra control traffic which may generate other frame collisions. 
Figure 20 Wireless network configuration, (a) cellular model (b) mesh model

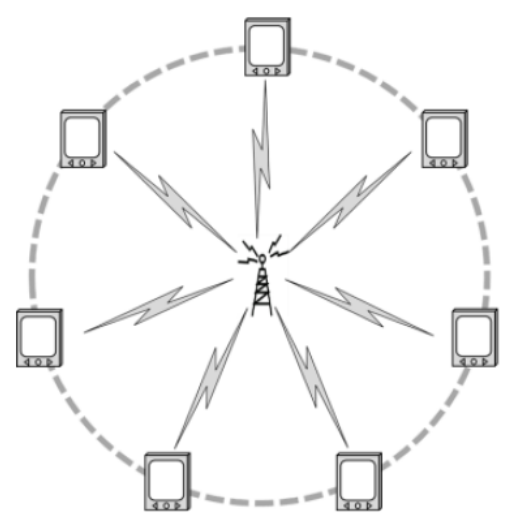

(a)

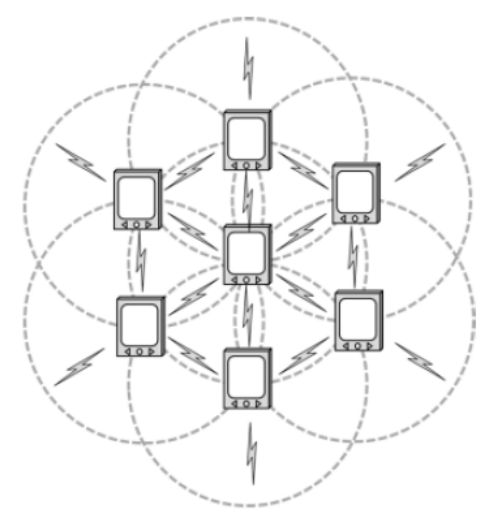

(b)

\subsubsection{ARM for mesh networking}

In order to solve the above problem, the active route maintenance (ARM) technology of maintaining a link was devised (Arutaki et al., 2007). The main idea of this ARM technology is to take advantage of data packet traffic to maintain an established link which conveys the traffic. If a data packet is received successfully in a path created by the routing protocol, namely, when a node receives data packets between Hello packet transmission interval, even if the receiving node cannot receive the Hello packet from transmitting node, it will maintain the link as illustrated in Figure 21(b). And also, when data packets are able to be transmitted successfully, the sending node maintains its link, even if it cannot receive the Hello packet from the transmitting node of the Hello; Figure 21(c) illustrates this occasion.

Figure 21 Active route maintenance

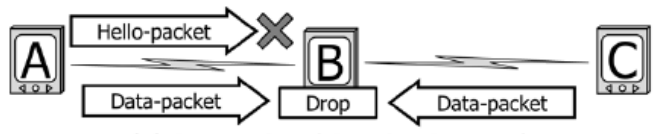

(a) Conventional Routing Protocol

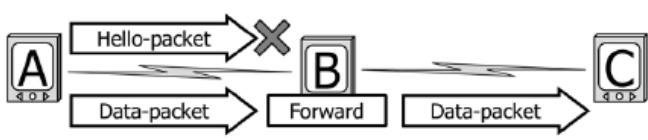

(b) Active Route Maintenance; Case 1

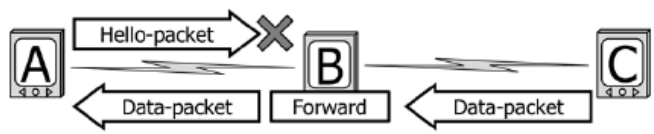

(c) Active Route Maintenance; Case 2

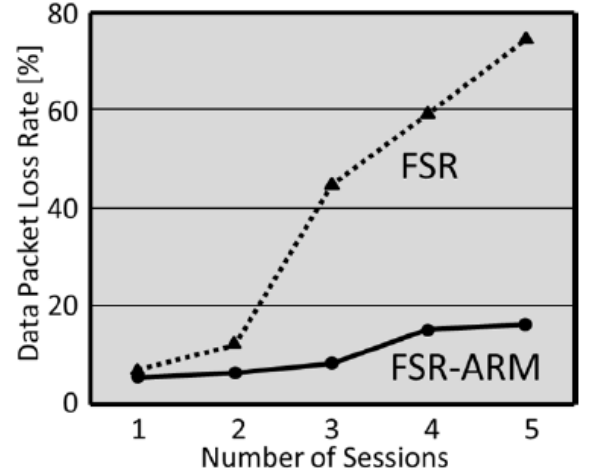

(d) Performance Comparison

In order to investigate the effect of this technology, comparative experiments of the proactive type routing protocol, i.e., fisheye state routing (FSR) (Gerla and Hong, 2002) and FSR-ARM which implemented the proposed ARM mechanism in FSR, were 
conducted. A Wi-Fi node broadcasts Hello packets of FSR at intervals of five seconds to discover adjacent nodes and to maintain established links. The established link by FSR is cut off, when a single Hello packet cannot be detected at the receiving node. In case of FSR-ARM, link cut-off occurs after three continuous losses of Hello packets. The Wi-Fi system of the experiment was IEEE802.11b. Five nodes were installed in both ends of the relay node, i.e., ten nodes totally, and were set to communicate via the relay node. As data packets, VoIP packets (UDP pay-load 372 byte, $50 \mathrm{~ms}$ intervals) specified by G.711 between the nodes of both ends were transmitted and received. The result of the error rate of data packets is illustrated in Figure 21(d), in which the number of UDP sessions varied from 1 to 5. The error rate of FSR-ARM is much better than that of the original FSR, so it is confirmed that the proposed ARM is effective for the better data forwarding and for less control traffic. This characteristics of the control system leads to the practical application of low RF power networking and thus to the frequency saving.

\subsubsection{DFF for mesh networking}

Other approaches to reduce the complexity of controlling wireless mesh-networks have been researched. One of the latest researches is depth first forwarding (DFF) protocol (Iwao et al., 2010) which does not broadcast control messages to discover destinations to deliver packets. Furthermore, DFF takes advantage of a data packet stream to find its destination; no destination discovery by control messages. The basic idea of DFF is as follows:

1 If the received data packet is for the receiving node, it is terminated at the node.

2 If the data packet is not for the receiving node, it is forwarded without knowing how to reach the final destination. Instead, the data packet is forwarded to one of the arbitrary child links connected to the node, which is randomly selected by the node.

3 If the node cannot find a child link anymore to forward the received data packet to, i.e., 'dead end', it returns the data packet to the original link from which the data packet was received. This operation is called 'backtrack'.

Figure 22 DFF protocol, (a) backtrack (b) loop detection

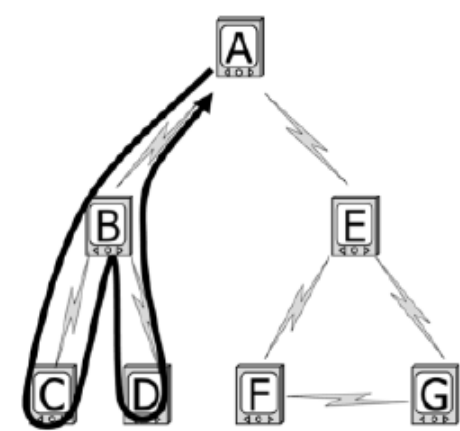

(a)

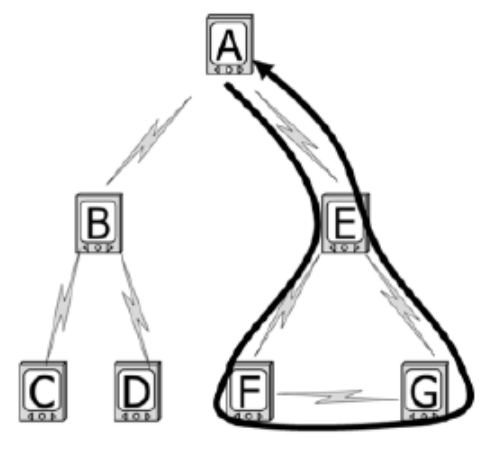

(b) 
Figure 22(a) illustrates the algorithm described above. It is necessary for all nodes in the network to tell any data packet apart from the others. Two fields in the data packet are checked by the receiving node for this purpose; one of them is an originator address $(\mathrm{OA})$, who generated the data packet, and the other is a sequence number (SN) which the originator assigned. The combination of the two fields makes it possible to identify the data packet. A new field is defined in a data packet for this protocol to perform a backtrack operation; return (RET) field. For example, the curved arrow in Figure 22(a) indicates this operation. The originator node-A generates a data packet, and forwards it to node-B. Node-B forwards the packet to node-C in case that the packet is not for node-B. Node-C recognises that the packet is not for itself, then returns it to node-B with setting the RET field, because node-C does not have any links other than with node-B; namely, node- $\mathrm{C}$ is the dead end. Node- $\mathrm{B}$ receives the packet from node- $\mathrm{C}$, and recognises that the packet is not brand new, i.e., the returned packet. Then node-B forwards the packet to node-D as an alternative recipient. The same operation is performed at node-D returning the packet to node- $\mathrm{B}$. At this moment, as node-B does not have any possible links to forward, node-B returns the packet to node-A.

The above operation resembles the depth first search algorithm for tree-structure networks. However, in case of wireless mesh networks, the network structure is not simply tree-shaped but 'meshed'. This mesh configuration constructs the looped structure, which may cause infinite forwarding operation along the loop. DFF protocol provides the following new mechanism to prevent the infinite looping. The curved arrow in Figure 22(b) explains the loop detection operation. When node-E receives a data packet from node-A, and recognises that the packet is not for itself, then node-E forward the data packet to node-F. Node-F also forwards it to node-G, then node- $G$ does so to node-E, too. Receiving this data packet, node-E detects that the RET field is not set in this data packet, even though it is not a brand new one for node-E. As this result, node-E decides that the packet is looped somewhere at its child nodes. Then node-E returns the data packet to node-A with setting the RET field. This indication informs node-A that there is not the final destination in its child nodes. Note that the above operation is described in a simplified manner for explanation purposes, and that DFF is furnished with much more sophisticated mechanisms. Refer to the request-for-comments (RFC) (Herberg et al., 2013) from IETF for more details.

This newly proposed mesh networking is also a candidate for future low RF-power communication to save frequencies. DFF was installed in some practical mesh networks for field trials according to the paper (Takahashi et al., 2011).

\section{Conclusions}

This paper introduced the direction and trends of research and development activities in Japan for saving natural resources such as energy and RF, which are focused more intensively after the East Japan megaquake. As one of the green ICT activities, the power saving of line IF in a router/switch was explained. Many smart grid projects in Japan have started their field trials; some of them were described in the paper. Finally, frequency saving technologies are introduced such as cognitive radio and mesh networking technologies. All of them will save natural resources more effectively than ever. 


\section{Acknowledgements}

The author would like to thank Professors M. Tsuru and M. Koeppen at Kyushu Institute of Technology for their generous advice regarding this manuscript. The special thanks should be dedicated to Professors K. Tsukamoto and Y. Fukuda at Kyushu Institute of Technology, who provided valuable materials to study.

\section{References}

Altintas, O., Ihara, Y., Kremo, H., Tanaka, H., Ohtake, M., Fujii, T., Yoshimura, C., Ando, K., Tsukamoto, K., Tsuru, M. and Oie, Y. (2012) 'Field tests and indoor emulation of distributed autonomous multi-hop vehicle-to-vehicle communications over TV white space', Proc. ACM 18th Mobicom, Istanbul, Turkey, August, pp.439-442.

ARRL (1989) The ARRL Handbook for the Radio Amateur, 66th ed., Chapt. 1, American Radio Relay League, Newington, CT, USA.

Arutaki, A., Matsumoto, A., Ito, T., Itoh, C., Ohteru, Y., Takahashi, N., Fujikawa, H., Suzuki, T. and Uesugi, N. (2007) 'Dependable wireless mesh network for next generation lifeline and business creation', Proc. 8th World Wireless Congress (WWC), Palo Alto, USA, 21-23 May.

Brown, M. and Sedano, R. (2004) Electricity Transmission A Primer, June, National Council on Electric Policy, Washington DC, USA.

Cabinet Office, Government of Japan (2012) Public Opinion Survey, April, in Japanese [online] http://www8.cao.go.jp/survey/h23/h23-life/index.html (accessed 30 May 2014).

Chubu Electric Power Company, Generating Stations to Households, in Japanese [online] http://www.chuden.co.jp/ryokin/information/chishiki/mame_hatsuden/index.html (accessed 30 May 2014)

Doyle, L. (2009) Essentials of Cognitive Radio, April, Cambridge University Press, Cambridge, UK.

Fukuda, Y., Kawamata, S., Ikenaga, T. and Oie, Y. (2012) 'Performance evaluation of the transmission control protocols with the dynamic link control scheme for power saving', Proc. IEEE GreenCom 2012, Besançon, France, November, pp.599-602.

Gerla, M. and Hong, X. (2002) Fisheye State Routing Protocol (FSR) for Ad Hoc Networks, IETF Draft, 17 June [online] http://tools.ietf.org/html/draft-ietf-manet-fsr-03 (accessed 30 May 2014).

Herberg, U., Cardenas, A., Iwao, T., Dow, M. and Cespedes, S. (2013) Depth-First Forwarding (DFF) in Unreliable Networks, IETF RFC6971, June [online] http://tools.ietf.org/html/rfc6971 (accessed 30 May 2014).

IEEE (2000) Link Aggregation, IEEE 802.3ad, March, Institute of Electrical and Electronics Engineers, New York, USA.

Iwao, T., Yamada, K., Yura, M., Nakaya, Y., Cárdenas, A.A., Lee, S. and Masuoka, R. (2010) 'Dynamic data forwarding in wireless mesh network', Proc. 1st IEEE International Conference on Smart Grid Communication (SmartGridCom), 4-6 October, Gaithersburg, Maryland, USA.

KEIDANREN (2013) A Proposal for Future Energy Policy, 15 October [online] https://www.keidanren.or.jp/en/policy/2013/089.html (accessed 30 May 2014).

Kimura, O. (2010) Japanese Top Runner Approach for Energy Efficiency Standards, SERC Discussion Paper: SERC09035 [online] http://www.denken.or.jp/en/serc/research_re/download/09035dp.pdf (accessed 30 May 2014).

Ministry of Economy, Trade and Industry (2010) Energy in Japan 2010, March [online] http://www.enecho.meti.go.jp/about/pamphlet/pdf/energy_in_japan2010_en.pdf (accessed 30 May 2014). 
Ministry of Internal Affairs and Communications (2008) Report on Study Group on ICT Policies against Global Warming, April [online] http://www.soumu.go.jp/main sosiki/joho tsusin/ policyreports/chousa/ict_globalwarming/pdf/0804_h1.pdf (in Japanese) (accessed 30 May 2014).

Ministry of Internal Affairs and Communications (2010) Report on the Vision for New Utilization of Radio Frequencies, July, in Japanese [online] http://www.soumu.go.jp/main_content/000079911.pdf (accessed 30 May 2014).

Ministry of Internal Affairs and Communications (2011) 2011 White Paper on Information and Communications in Japan, in Japanese [online] http://www.soumu.go.jp/johotsusintokei/whitepaper/ja/h23/pdf/23honpen.pdf (accessed 30 May 2014).

Ministry of Internal Affairs and Communications (2013) Ordinance of the Ministry of Internal Affairs and Communications No.105, 10 December, in Japanese [online] http://law.e-gov.go.jp/htmldata/S25/S25F30901000014.html (accessed 30 May 2004)

Mitsui Fudosan Co. Ltd. (2014) Press release, 24 April, in Japanese [online] http://www.mitsuifudosan.co.jp/corporate/news/2014/0424_03/index.html (accessed 30 May 2014).

Nikkan Kogyo Shimbun, Ltd. (2013) Smart House Trial in Ishikawa Prefecture, Nikkan Kogyo Shimbun Business Line, 2 October, in Japanese [online] http://www.nikkan.co.jp/toku/smartglid/sg20131002-12n-415ps.html (accessed 30 May 2014)

NTT Data Institute of Management Consulting, Inc. (2013) Report on Infrastructure Improvement for Information Economy Society of Japan (Investigation of Energy Consumption of IT Equipment), February, in Japanese [online] http://www.meti.go.jp/meti_lib/report/2013fy/E002741.pdf (accessed 30 May 2014).

Suzuki, H. (2012) Green Curtain; Social Acceptance Process of its Policy, JICHISOKEN, The Japan Research Institute for Local Government, in Japanese, September, Vol. 407, pp.71-86.

Takahashi, Y., Kawashima, K., Nakaya, Y. and Ichikawa, T. (2011) 'Network technology supporting an intelligent society: WisReed', Fujitsu Sci. Tech. J., October, Vol. 47, No. 4, pp.474-481.

Teranishi, A., Suzuki, S., Shizuno, K., Asada, M., Sugiyama, H. and Yokoyama, H. (2012) 'Estimation of transit time in terahertz oscillating resonant tunnelling diodes with graded emitter and thin barriers', IEICE Trans. on Electronics, Vol. E95-C, No. 3, pp.401-407.

The Federation of Electric Power Companies (2010) Present State of Electric Utility Industry 2011, Brochure, November, in Japanese.

The Federation of Electric Power Companies (2011) Energy Balance of Major Countries, Graphical Flip-chart of Nuclear \& Energy Related Topics, pp.1-11, in Japanese [online] http://www.fepc.or.jp/library/publication/pamphlet/nuclear/zumenshu/digital/ (accessed 30 May 2014)

The Federation of Electric Power Companies (2013) Composition Ratio of Electric Power Generation, 17 May, in Japanese [online] http://www.fepc.or.jp/about_us/pr/sonota/_icsFiles/afieldfile/2013/05/17/kouseihi_2012.pdf (accessed 30 May 2014)

Tokyo Electric Power Company (2014) Installation of Smart Meters, Press release, 2 April, in Japanese [online] http://www.tepco.co.jp/cc/press/2014/1235281_5851.html (accessed 30 May 2014). 